\title{
NOVEL COCRYSTALS AND EUTECTICS OF THE ANTIPROTOZOAL TINIDAZOLE: MECHANOCHEMICAL SYNTHESIS, COCRYSTALLIZATION AND CHARACTERIZATION
}

Octavio E. Fandiño, Lucía Reviglio, Yamila Garro Linck, Gustavo A. Monti, Marina M. Marcos Valdez, Sonia N. Faudone, Mino R. Caira, Norma R. Sperandeo

\section{SUPPLEMENTARY INFORMATION INDEX}

\begin{tabular}{|c|c|}
\hline DESCRIPTION & $\begin{array}{l}\text { Page } \\
\mathrm{N}^{\circ} \text {. }\end{array}$ \\
\hline SCXRD experimental details. & 3 \\
\hline 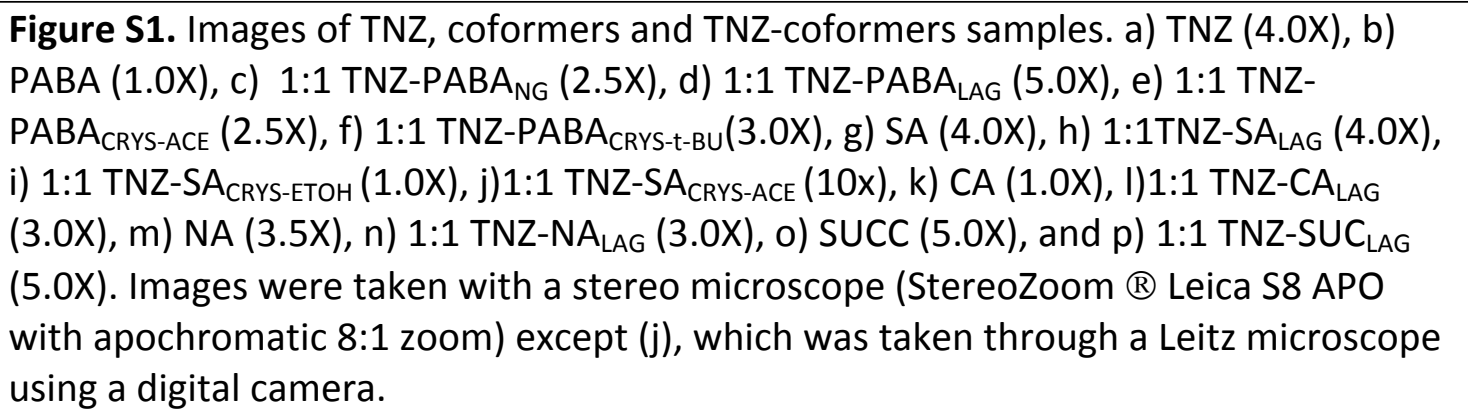 & 4 \\
\hline $\begin{array}{l}\text { Table S1. Melting points (capillary method) for tinidazole, coformers and samples } \\
\text { tested. }\end{array}$ & 5 \\
\hline $\begin{array}{l}\text { Figure S2. Powder X-ray patterns of the following samples and experiments (in 1:1 } \\
\text { molar ratio for binary systems): (a) TNZ, (b) SUC, (c) PM, and (d) TNZ-SUC } C_{\text {LAG. }}\end{array}$ & 6 \\
\hline $\begin{array}{l}\text { Figure S3. Powder X-ray patterns of the following samples and experiments (in 1:1 } \\
\text { molar ratio for binary systems): (a) TNZ, (e) NA, (f) PM, and (g) TNZ-NA } A_{\text {LAG. }}\end{array}$ & 7 \\
\hline $\begin{array}{l}\text { Table S2. Crystal data, data-collection parameters and refinement details for TNZ- } \\
\text { PABA }_{\text {CRYS-TBU. }}\end{array}$ & 8 \\
\hline Table S3. Crystal data, data-collection parameters and refinement details for TNZ-SA. & 9 \\
\hline $\begin{array}{l}\text { Figure S4. }{ }^{1} \mathrm{H} \text { NMR spectra of TNZ-PABA } \\
\text { deuterated chu at } t=0 \text { in closed NMR tube }(\mathrm{a}) \text {, } \\
\text { spectrum of TNZ-PABA with a few drops of acetone of crystallization }(b) \text { and }{ }^{1} \mathrm{H} N M R \\
\text { SRY-tBU at } t=20 \mathrm{~d} \text { in open NMR tube (c). }\end{array}$ & 10 \\
\hline $\begin{array}{l}\text { Figure S5. }{ }^{13} \text { C CPMAS NMR spectra of the following samples and experiments (in 1:1 } \\
\text { molar ratio for binary systems): (a) TNZ, (b) SUC, (c) PM, and (d) TNZ-SUC } C_{\text {LAG. }}\end{array}$ & 11 \\
\hline $\begin{array}{l}\text { Figure S6. }{ }^{13} \mathrm{C} \text { CPMAS NMR spectra of the following samples and experiments (in 1:1 } \\
\text { molar ratio for binary systems): (a) TNZ, (b) NA, (c) PM, and (d) TNZ-NA } A_{\text {LAG. }}\end{array}$ & 12 \\
\hline $\begin{array}{l}\text { Figure S7. DRIFT spectra of the following samples and experiments (in 1:1 molar ratio } \\
\text { for binary systems): (a) TNZ, (b) PABA, (c) TNZ-PABA } \text { LAG. }\end{array}$ & 13 \\
\hline $\begin{array}{l}\text { Figure S8. DRIFT spectra of the following samples and experiments (in 1:1 molar ratio } \\
\text { for binary systems): (a) TNZ, (b) SA, and (c) TNZ-SAL LAG. }\end{array}$ & 14 \\
\hline $\begin{array}{l}\text { Figure S9. DRIFT spectra of the following samples and experiments (in 1:1 molar ratio } \\
\text { stoichiometry): (a) TNZ, (b) CA and (c) TNZ-CA } A_{L A G}\end{array}$ & 15 \\
\hline $\begin{array}{l}\text { Figure S10. FTIR spectra of the following samples and experiments (in 1:1 molar ratio } \\
\text { for binary systems): (a) TNZ, (b) SUC, and (c) TNZ-SUC } C_{\text {LAG. }}\end{array}$ & 16 \\
\hline
\end{tabular}




\begin{tabular}{|c|c|}
\hline $\begin{array}{l}\text { Figure S11. FTIR spectra of the following samples and experiments (in 1:1 molar ratio } \\
\text { for binary systems): (a) TNZ, (b) NA, and (c) TNZ-NA } A_{\text {LAG. }}\end{array}$ & 17 \\
\hline $\begin{array}{l}\text { Figure S12. DSC and TG curves (heating rate of } 10^{\circ} \mathrm{C} / \mathrm{min} \text {, flowing } \mathrm{N}_{2} \text { at } 50 \mathrm{~mL} / \mathrm{min} \text { ) } \\
\text { for: a) TNZ; b) SUC; c) 1:1 TNZ-SUC PM; d) TNZ-SUC CAG. }\end{array}$ & 18 \\
\hline $\begin{array}{l}\text { Figure S13. DSC and TG curves (heating rate of } 10^{\circ} \mathrm{C} / \mathrm{min} \text {, flowing } \mathrm{N}_{2} \text { at } 50 \mathrm{~mL} / \mathrm{min} \text { ) } \\
\text { for: a) TNZ; b) NA and c) TNZ -NA } A_{\text {LAG. }}\end{array}$ & 19 \\
\hline $\begin{array}{l}\text { Figure S14. Images for TNZ, coformers and their binary samples before (a) and after } \\
\left.\text { exposition (b) of light. Keys: 1) TNZ; 2) TNZ-PABA }{ }_{L A G} ; 3\right) \text { TNZ-PABA PM; 4) TNZ-CA }{ }_{L A G} \\
\text { 5) TNZ-CA PM; 7) TNZ-SA } \\
N_{L A G} \text {; 8) TNZ-SA PM; 9)TNZ-SUC } C_{L A G} \text {;0) TNZ-SUC PM; 11) TNZ- } \\
\text { and TNZ-NA PM. }\end{array}$ & 20 \\
\hline $\begin{array}{l}\text { Table S4. }{ }^{13} \mathrm{C} \text { CP/MAS chemical shifts (in ppm) for tinidazole and its cocrystals with } p \text { - } \\
\text { aminobenzoic acid, salicylic acid and citric acid. }\end{array}$ & 21 \\
\hline Table S5. $\mathrm{pH}$ values after equilibrium solubilities experiments $\left(37^{\circ} \mathrm{C}\right)$ & 23 \\
\hline
\end{tabular}




\section{SCXRD experimental details}

For both crystals an important feature of the X-ray analyses was to establish the nature of these crystalline phases, specifically whether they are cocrystals or salts. This required careful inspection of difference electron density maps at an advanced stage of the refinement to locate hydrogen atoms unequivocally and this was achieved without any difficulty. While the X-ray analysis of the unsolvated phase TNZ-SA presented no serious challenge, that of TNZ-PABA CRYS-t-Bu $_{\text {. }}$ required extensive least-squares refinement in order to obtain an acceptable final model. This was due to the presence of solvent $(t-\mathrm{BuOH})$ in low stoichiometric ratio, with the molecule disordered over two positions at an inversion center. The presence of discrete peaks for the solvent nonhydrogen atoms eventually enabled their modeling, although it was necessary to introduce several distance restraints and to treat these atoms isotropically with a global free variable for their displacement parameters owing to the low solvent site-occupancy. For both crystal structures, however, all non-H atoms of TNZ and the respective molecules used for co-crystallization (PABA and SA) were successfully refined anisotropically. After location of all $\mathrm{H}$ atoms in electron density maps, they were generally added in idealized positions in a riding model with isotropic displacement parameters in the range 1.2-1.5 times those of their parent atoms. 
Figure S1

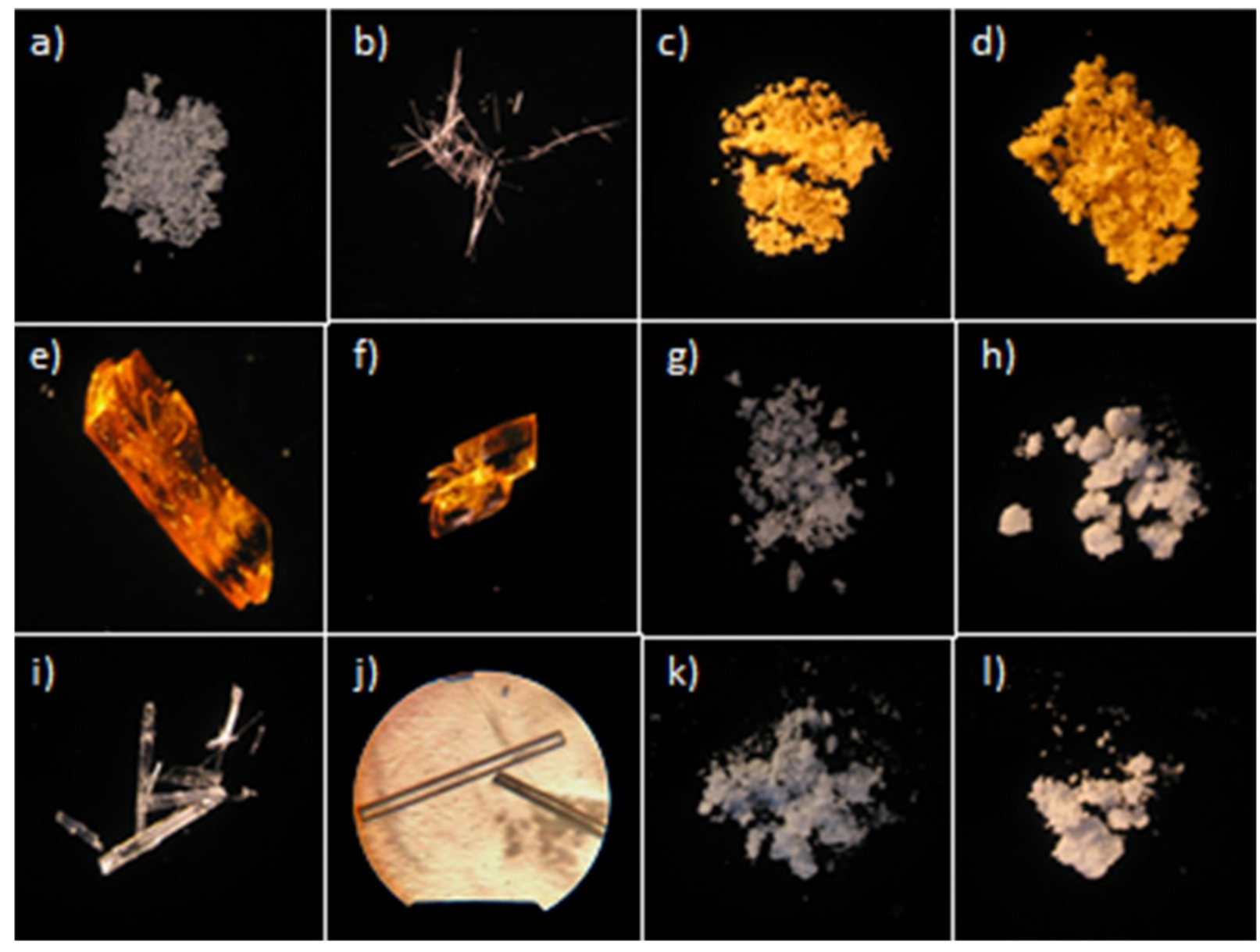




\section{Table S1}

\begin{tabular}{|c|c|}
\hline Sample & m.p., ${ }^{\circ}{ }^{\circ} \mathrm{C}$ \\
\hline $\mathrm{TNZ}-\mathrm{PABA} \mathrm{NG}_{\mathrm{NG}}$ & 132.0 \\
\hline \multirow[t]{2}{*}{$T^{T N Z-P A B A} A_{L A G}$} & 112.8 \\
\hline & 134.0 \\
\hline \multirow[t]{2}{*}{ TNZ-PABA $_{\text {CRYS-ACE }}$} & 113.1 \\
\hline & 133.5 \\
\hline \multirow[t]{2}{*}{ TNZ-PABA ${ }_{C R Y S-t B U}$} & 113.6 \\
\hline & 132.8 \\
\hline TNZ-SA & 118.0 \\
\hline TNZ-SA $A_{C R Y S-E T O H}$ & 118.4 \\
\hline $\mathrm{TNZ}^{-C A_{L A G}}$ & 121.6 \\
\hline TNZ-SUC $\mathrm{LAG}_{\mathrm{LAG}}$ & 106.0 \\
\hline TNZ-NA $A_{L A G}$ & 102.0 \\
\hline TNZ & 126.0 \\
\hline PABA & 188.0 \\
\hline SA & 158.0 \\
\hline $\mathrm{CA}$ & 153.2 \\
\hline SUC & 184.1 \\
\hline NA & 128.0 \\
\hline
\end{tabular}

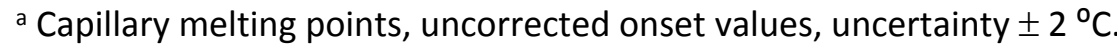


Figure S2

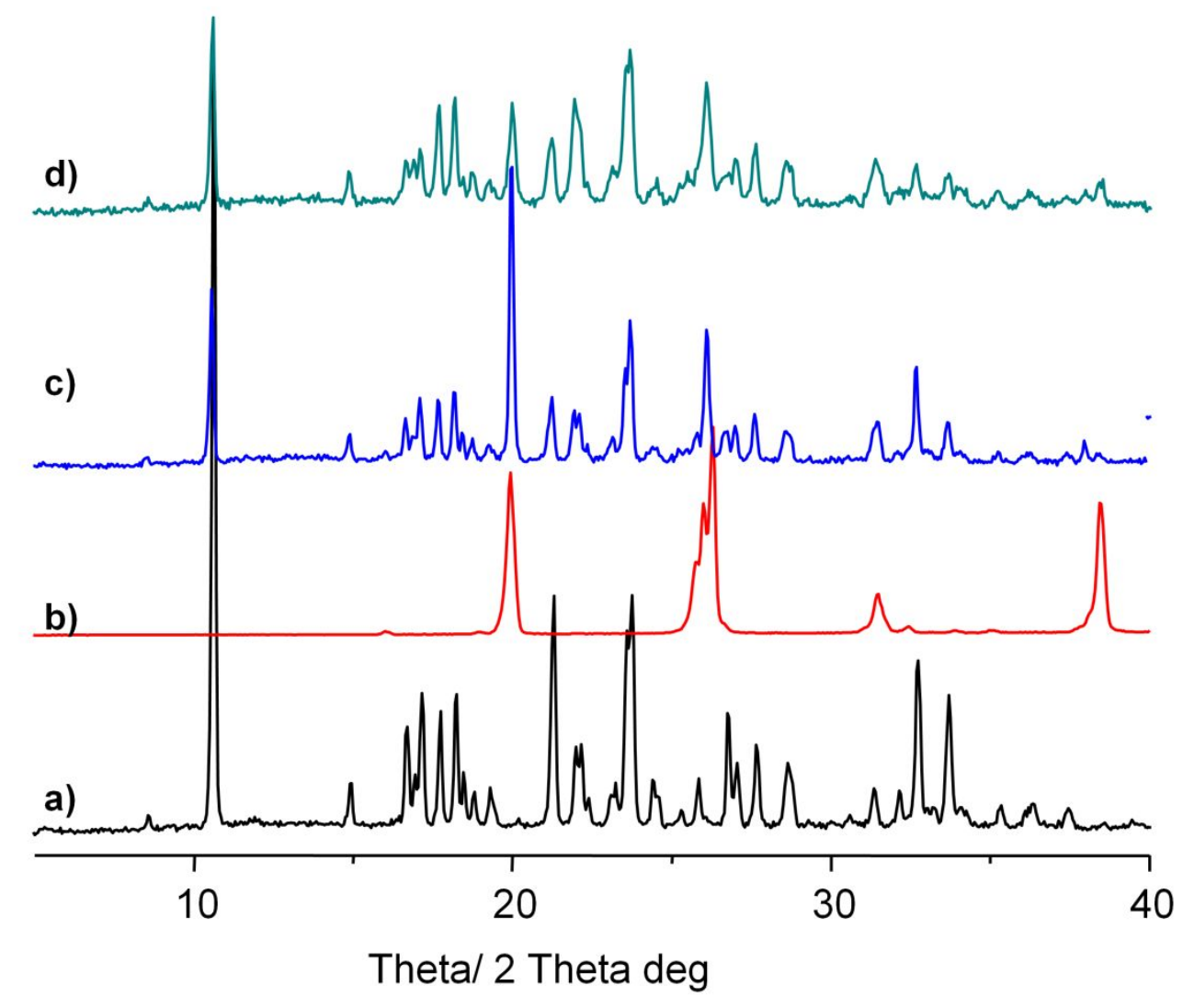


Figure S3

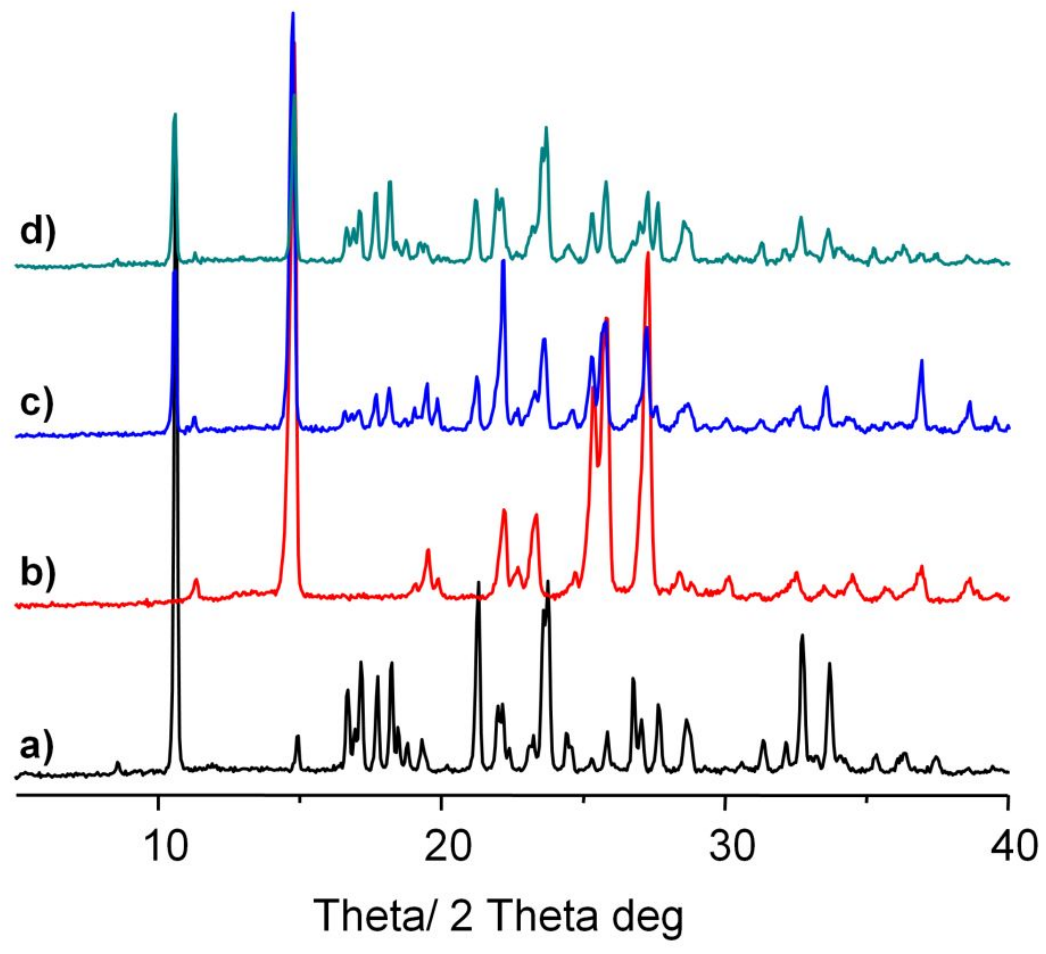


Table S2

\begin{tabular}{|l|l|}
\hline Moiety formula & $\mathrm{C}_{8} \mathrm{H}_{13} \mathrm{~N}_{3} \mathrm{O}_{4} \mathrm{~S} \cdot \mathrm{C}_{7} \mathrm{H}_{7} \mathrm{NO}_{2} \cdot 0.4\left(\mathrm{C}_{4} \mathrm{H}_{10} \mathrm{O}\right)$ \\
\hline Formula mass $\left(\mathrm{g} \mathrm{mol}^{-1}\right)$ & 414.06 \\
\hline Crystal dimensions $(\mathrm{mm})$ & $0.27,0.15,0.14$ \\
\hline Crystal system & Monoclinic \\
\hline Space group & $P 2 / n(\mathrm{No} .14)$ \\
\hline$a(\AA), b(\AA), c(\AA), \beta\left(^{\circ}\right), V\left(\AA^{3}\right)$ & $5.8529(4), 14.2198(11), 24.304(2), 90.179(2)$, \\
& $2022.8(3)$ \\
\hline$Z$ & 4 \\
\hline$D_{\mathrm{c}}\left(\mathrm{g} \mathrm{cm}^{-3}\right)$ & 1.360 \\
\hline$F_{000}$ & 875 \\
\hline$\mu\left(\mathrm{mm}^{-1}\right)$ & 0.203 \\
\hline$\lambda(\AA)$ & 0.71073 \\
\hline$T(\mathrm{~K})$ & $173(2)$ \\
\hline $2 \theta_{\text {max }}\left(^{\circ}\right)$ & 55.2 \\
\hline Reflections measured/unique/R $\mathrm{R}_{\text {int }}$ & $28762 / 4688 / 0.0486$ \\
\hline Reflections with I $>2 \sigma(\mathrm{I})$ & 3764 \\
\hline Parameters/restraints & $263 / 9$ \\
\hline$R 1, w R 2,[\mathrm{I}>2 \sigma(\mathrm{I})]$ & $0.0580,0.1783$ \\
\hline Goodness-of-fit $(\mathrm{S})$ & 1.046 \\
\hline CCDC no. & 1960455 \\
\hline
\end{tabular}


Table S3

\begin{tabular}{|l|l|}
\hline Moiety formula & $\mathrm{C}_{8} \mathrm{H}_{13} \mathrm{~N}_{3} \mathrm{O}_{4} \mathrm{~S} \cdot \mathrm{C}_{7} \mathrm{H}_{6} \mathrm{O}_{3}$ \\
\hline Formula mass $\left(\mathrm{g} \mathrm{mol}{ }^{-1}\right)$ & 385.39 \\
\hline Crystal dimensions $(\mathrm{mm})$ & $0.42,0.29,0.18$ \\
\hline Crystal system & Monoclinic \\
\hline Space group & $P{ }_{1} / c($ No. 14$)$ \\
\hline$a(\AA), b(\AA), c(\AA), \beta\left(^{\circ}\right), V\left(\AA^{3}\right)$ & $5.7785(2), 14.5717(6), 20.6134(8), 92.3510(10)$, \\
& $1734.24(12)$ \\
\hline$Z$ & 4 \\
\hline$D_{c}\left(\mathrm{~g} \mathrm{~cm}^{-3}\right)$ & 1.476 \\
\hline$F_{000}$ & 808 \\
\hline$\mu\left(\mathrm{mm}^{-1}\right)$ & 0.231 \\
\hline$\lambda(\AA)$ & 0.71073 \\
\hline$T(\mathrm{~K})$ & $173(2)$ \\
\hline $2 \theta_{\text {max }}\left({ }^{\circ}\right)$ & 56.8 \\
\hline Reflections measured/unique/ $\mathrm{R}_{\text {int }}$ & $28676 / 4347 / 0.0335$ \\
\hline Reflections with I $>2 \sigma(\mathrm{I})$ & 3738 \\
\hline Parameters/restraints & $239 / 0$ \\
\hline$R 1, w R 2,[\mathrm{I}>2 \sigma(\mathrm{I})]$ & $0.0332,0.0880$ \\
\hline Goodness-of-fit (S) & 1.045 \\
\hline CCDC no. & 1960462 \\
\hline
\end{tabular}


Figure S4
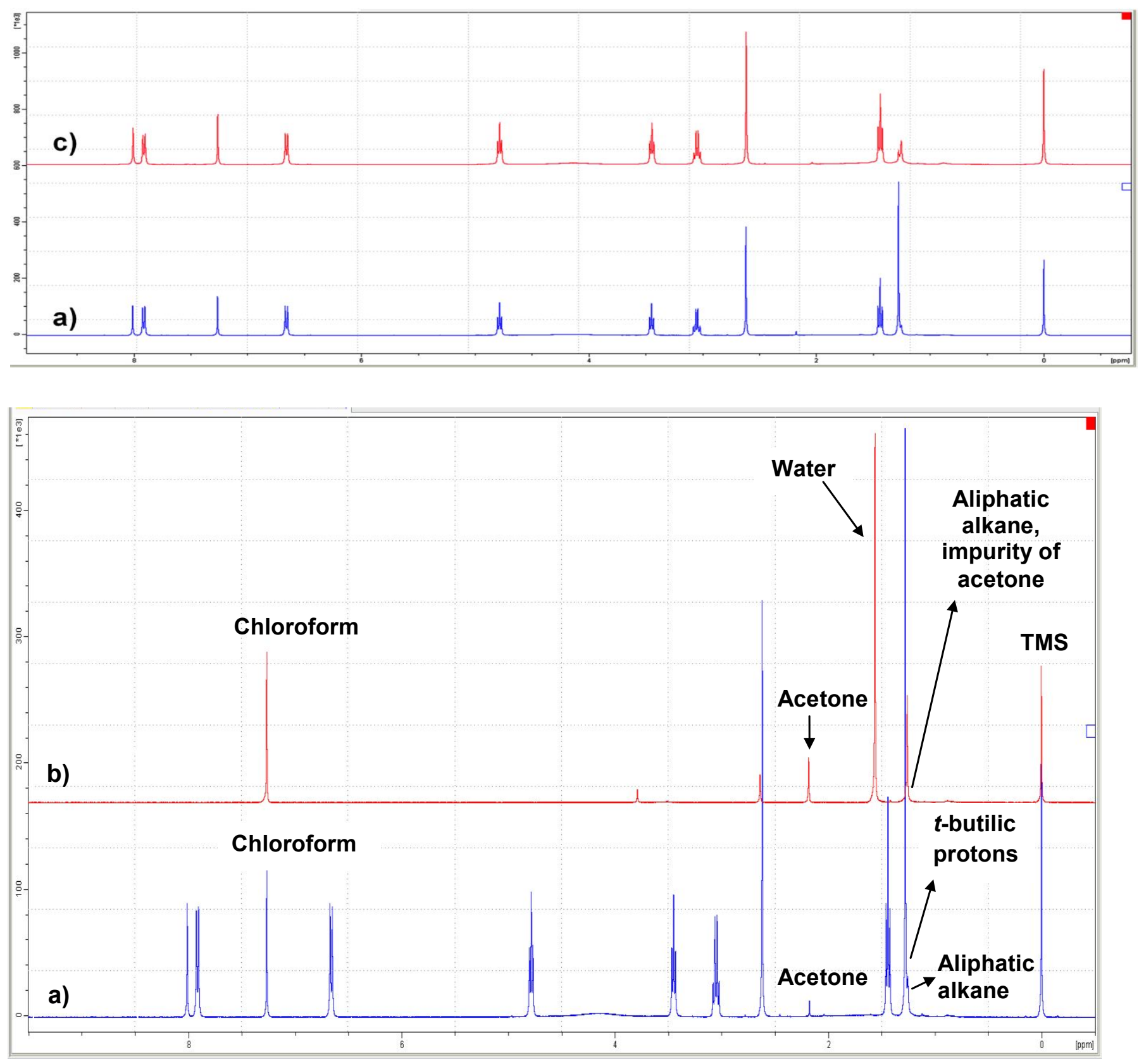
Figure S5

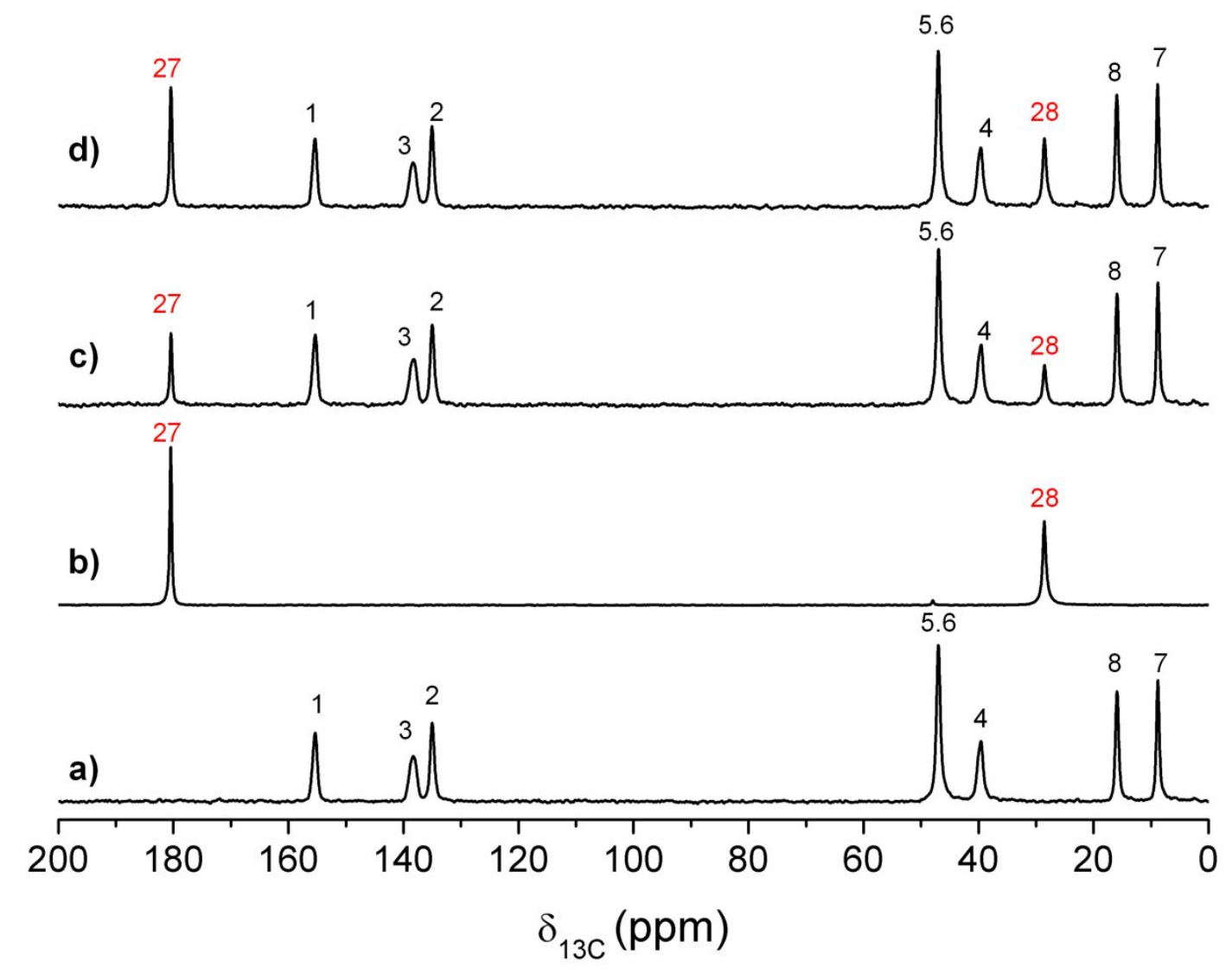


Figure S6
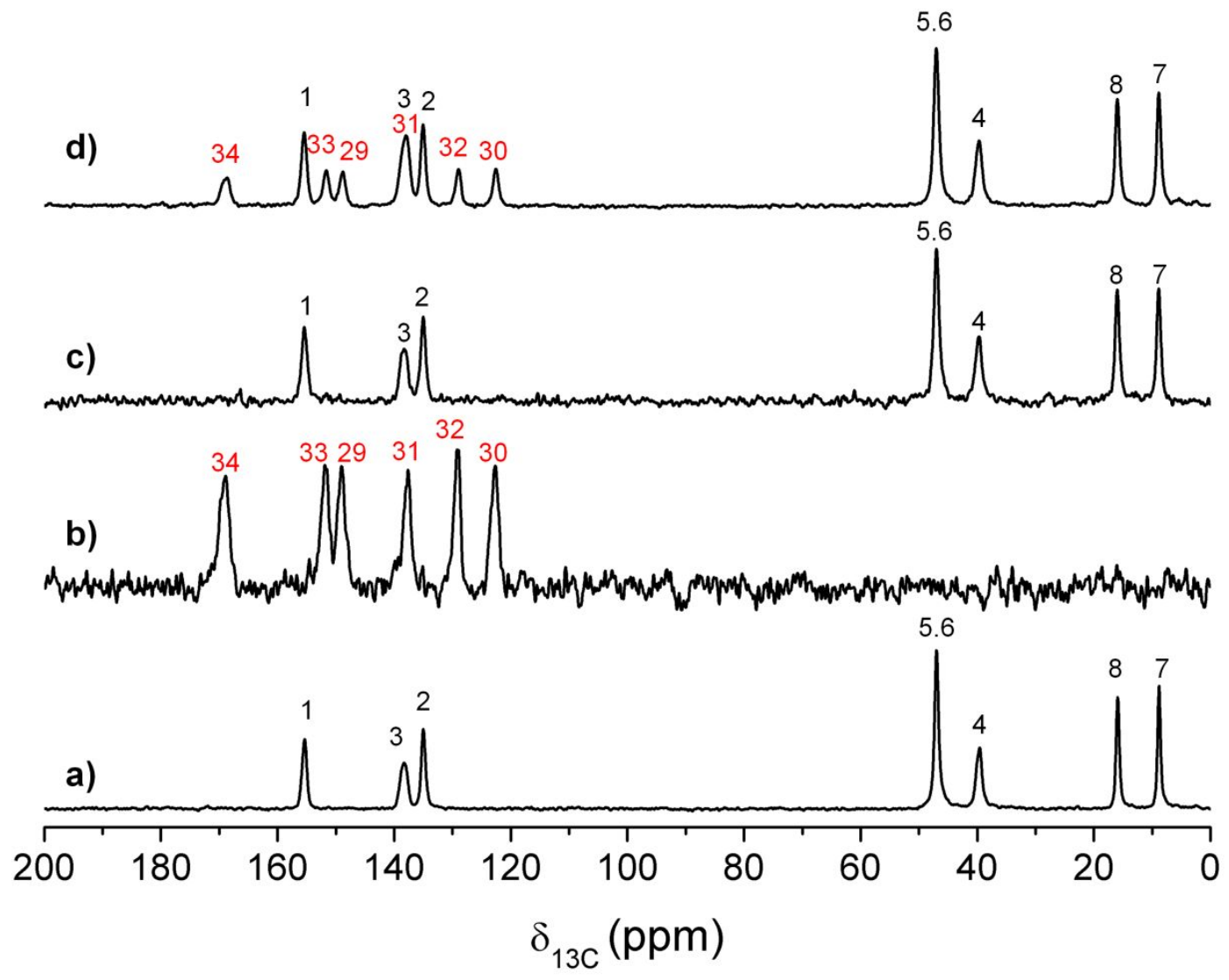
Figure S7

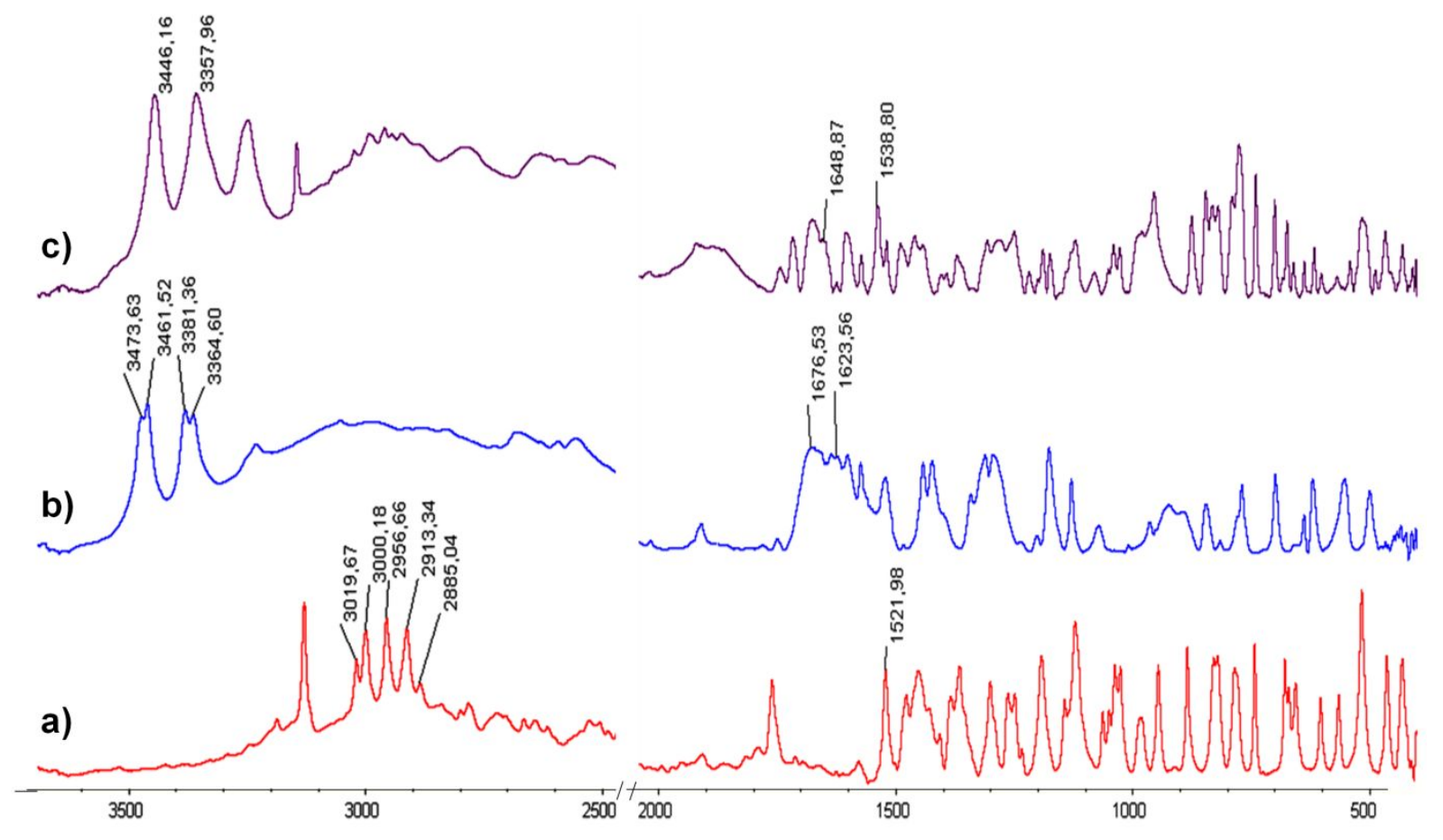


Figure S8
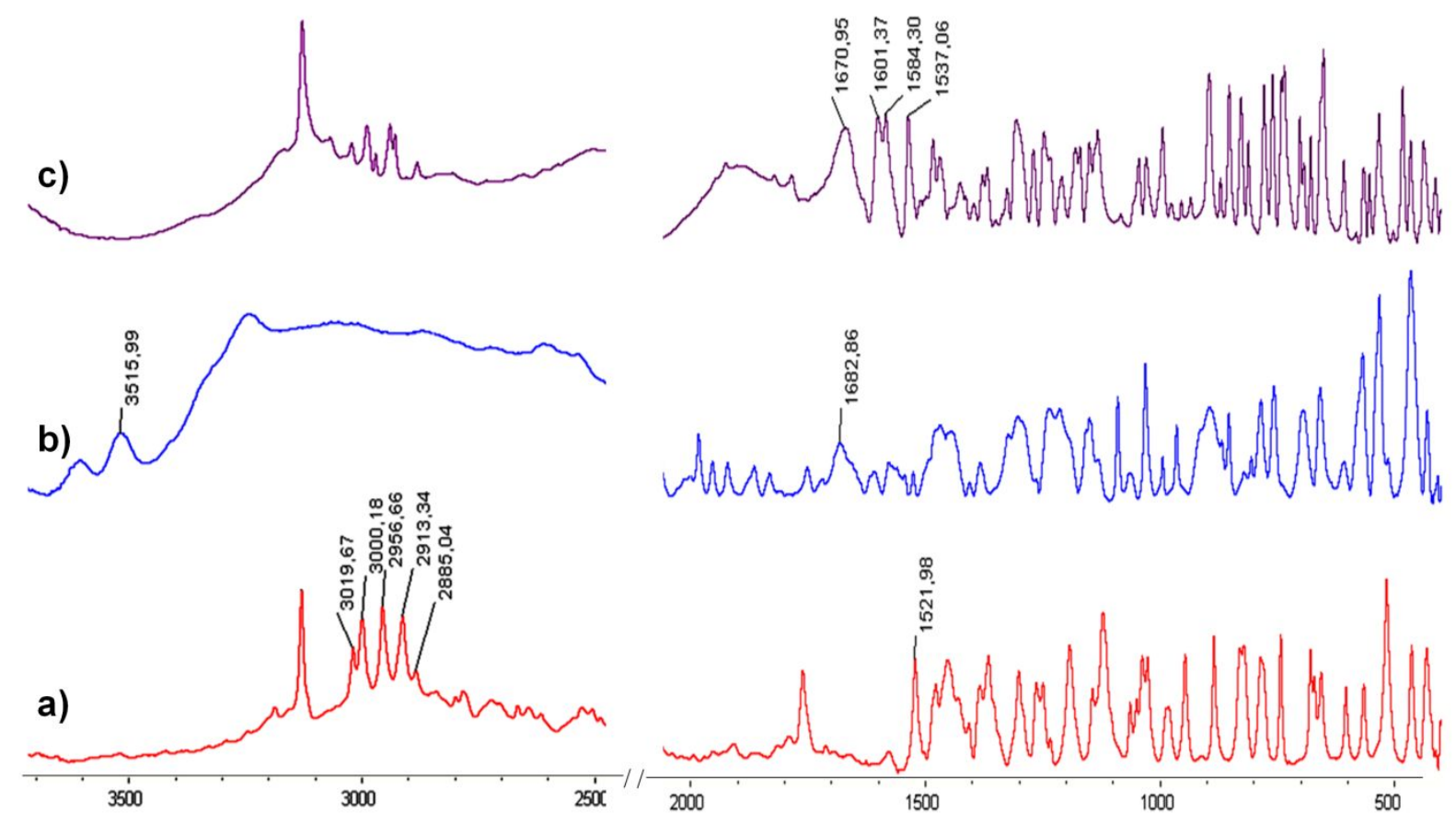
Figure S9

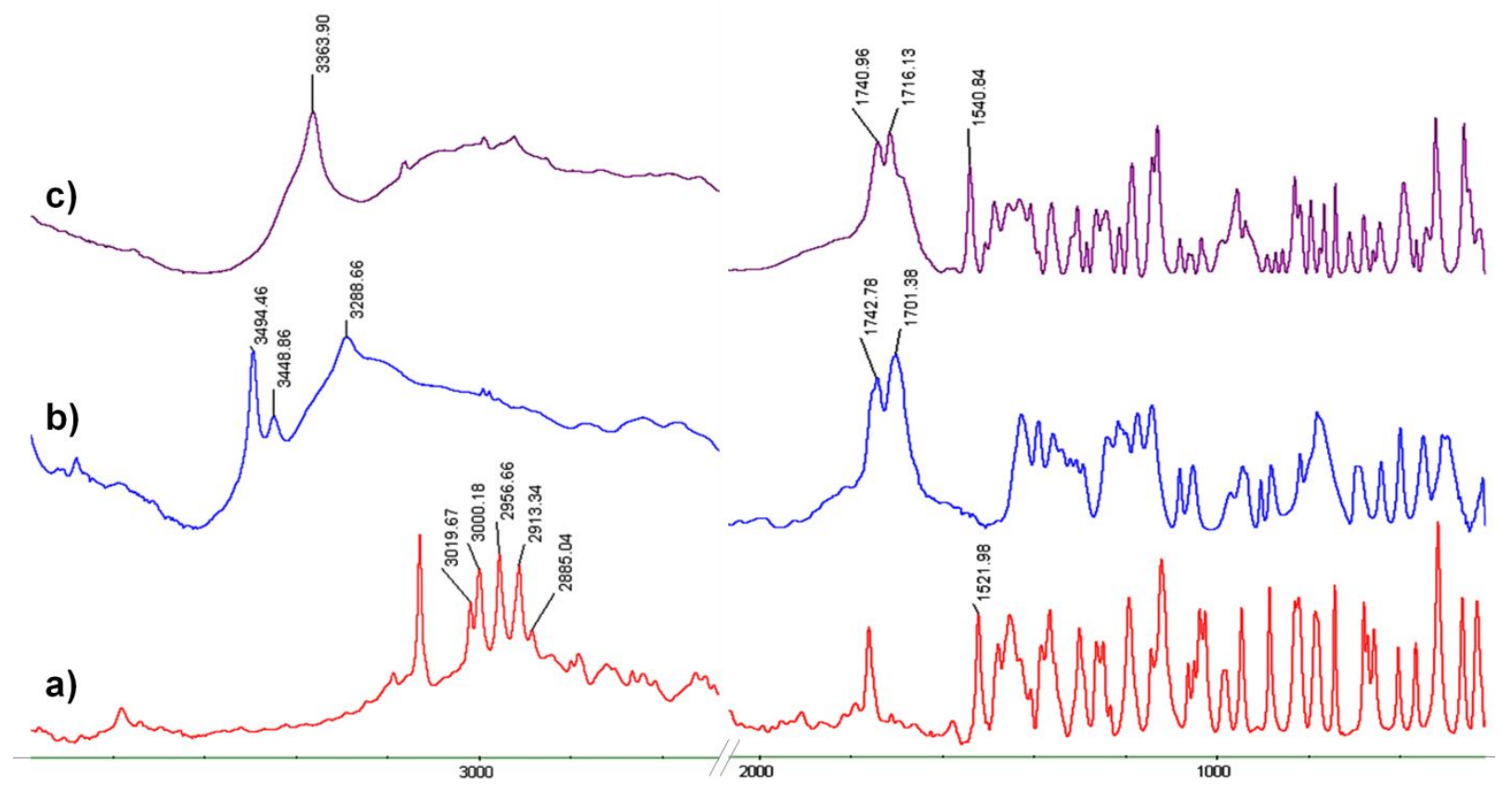


Figure S10

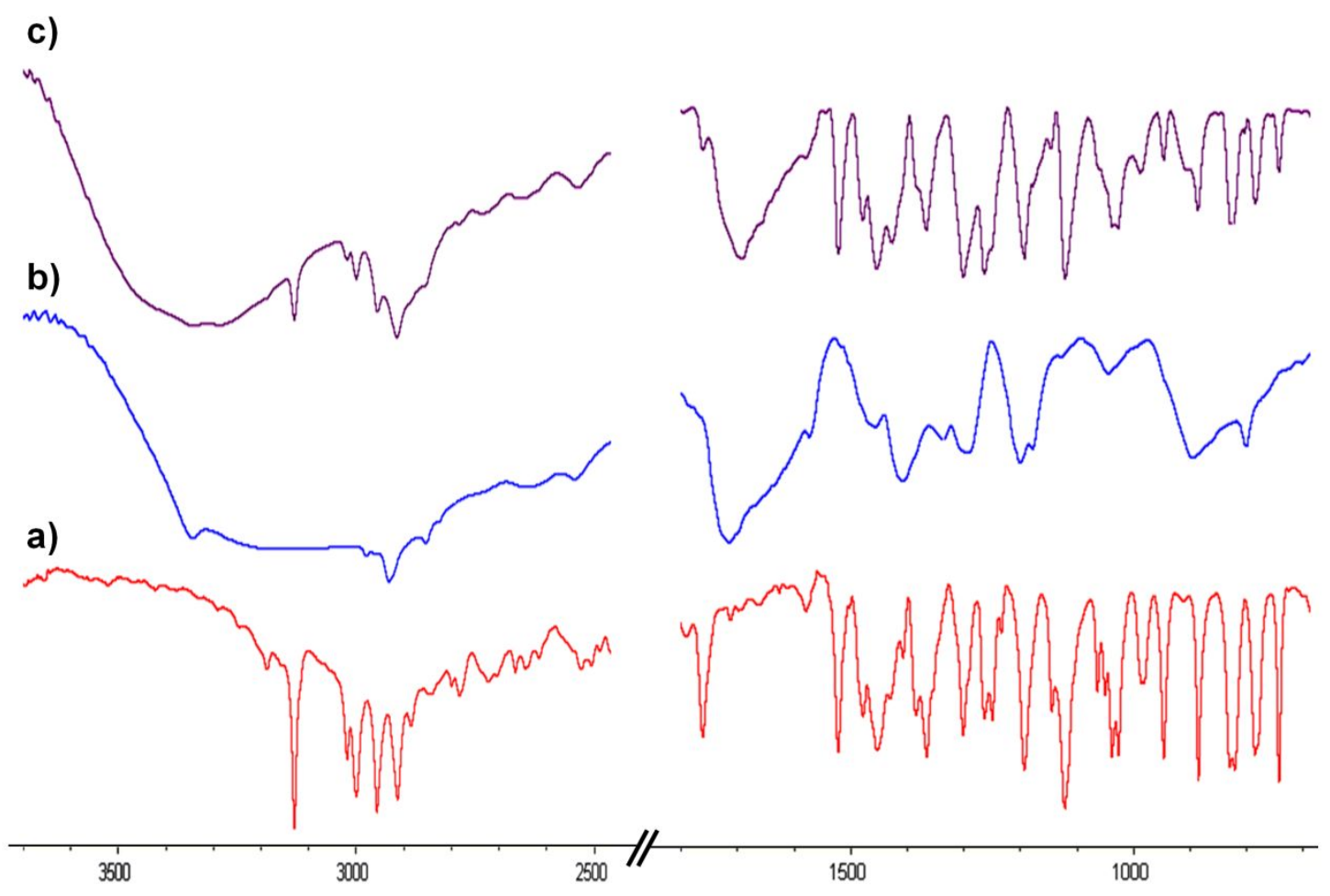


Figure S11

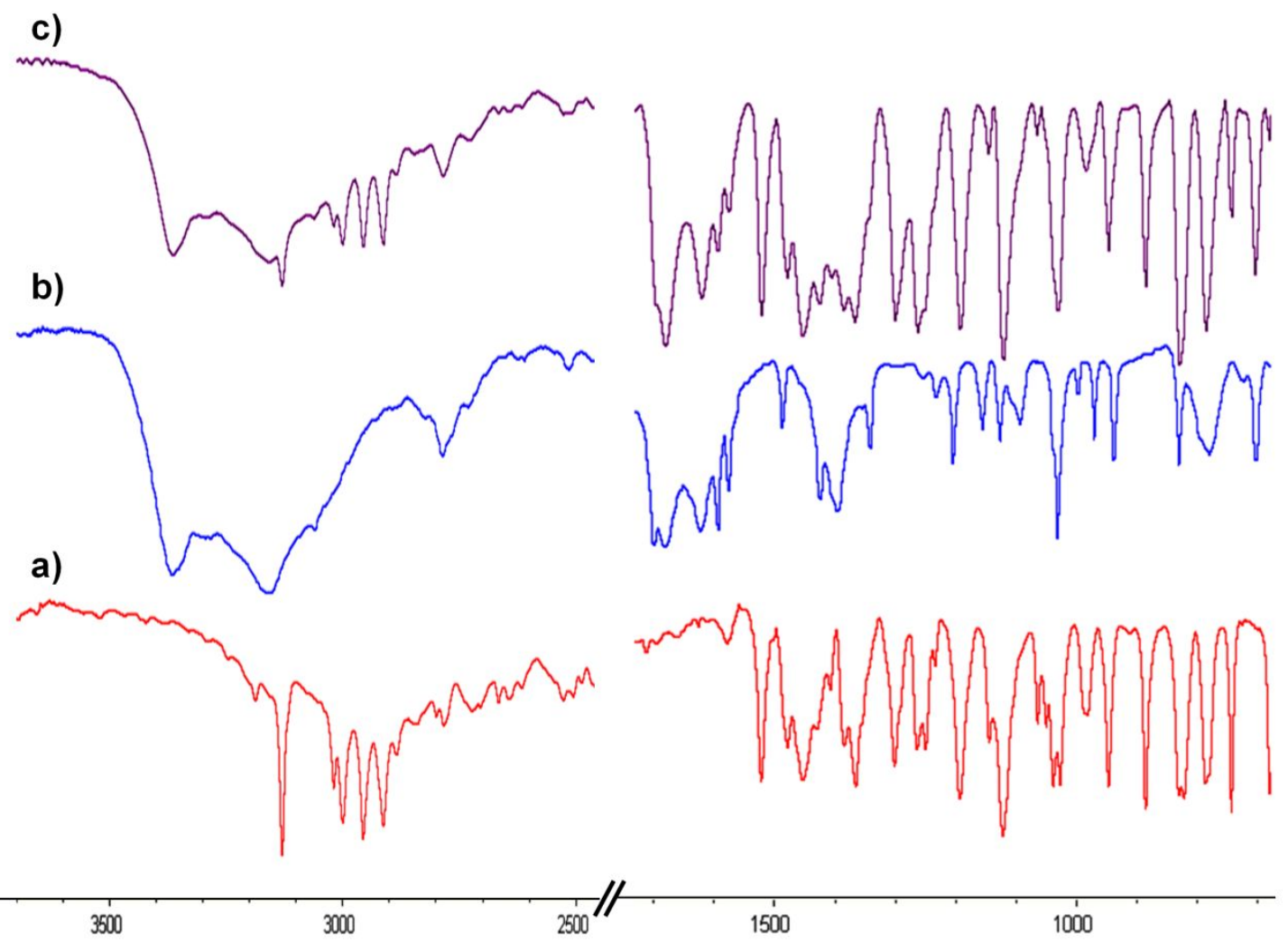


Figure S12

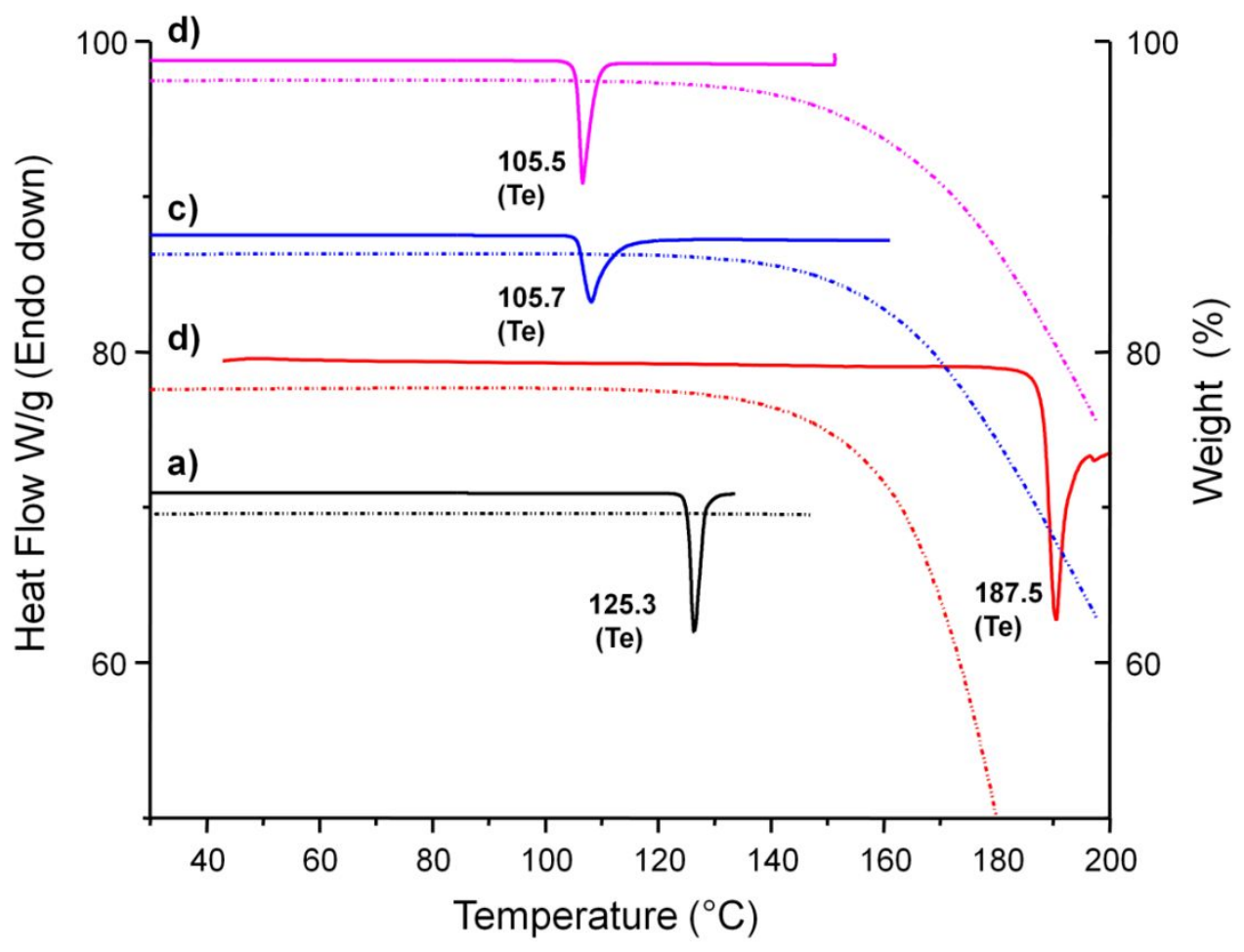


Figure S13

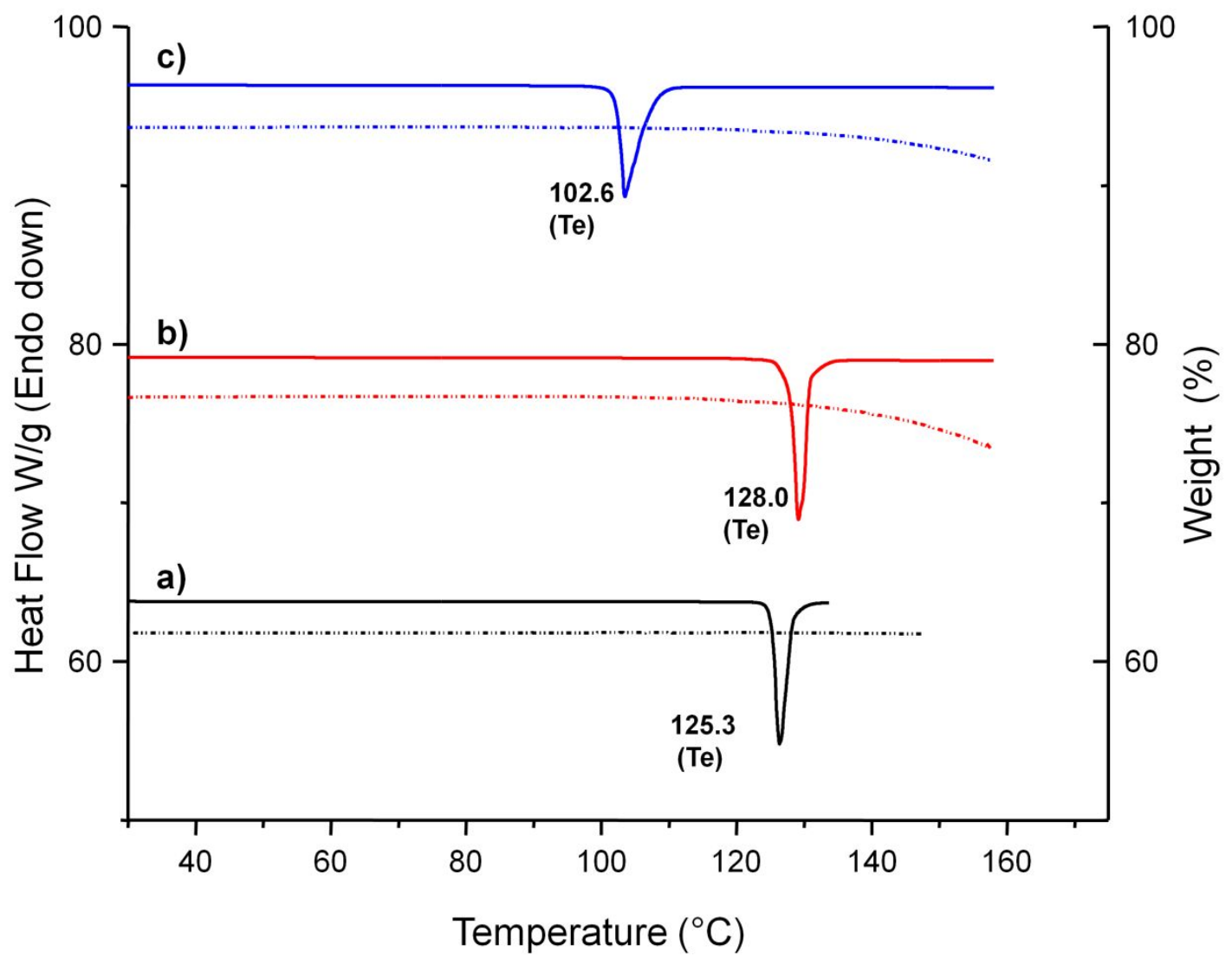


Figure S14

a)
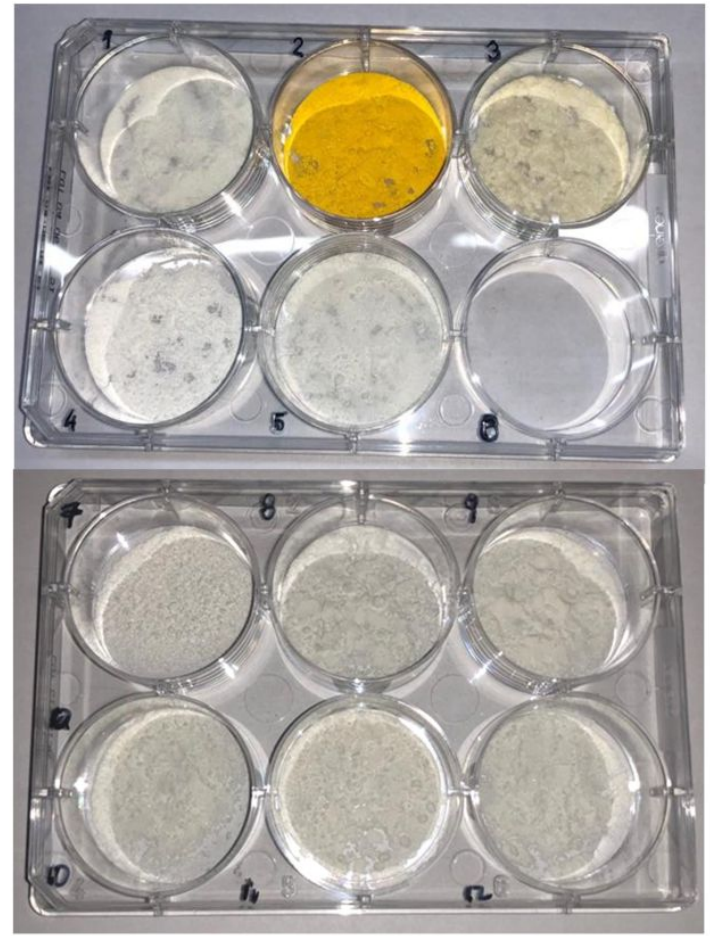

b)
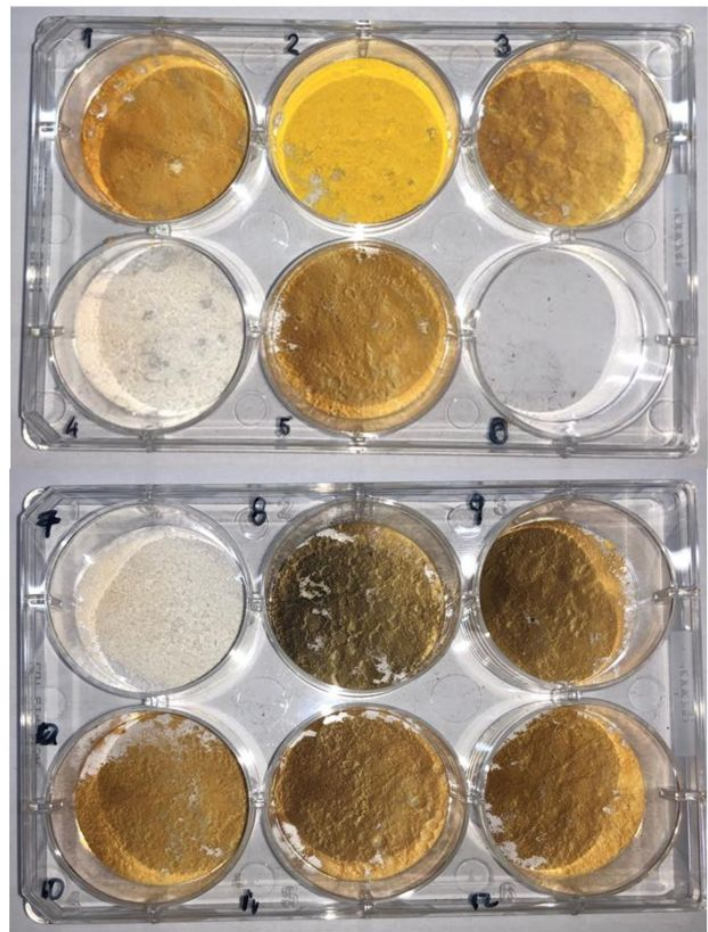
Table S4

\begin{tabular}{|c|c|c|c|c|c|c|c|c|c|c|c|c|}
\hline \multirow{2}{*}{$\begin{array}{c}\text { Carbon } \\
\text { atom }\end{array}$} & \multirow[b]{2}{*}{ TNZ } & \multirow[b]{2}{*}{ PABA } & \multirow[b]{2}{*}{ SA } & \multirow[b]{2}{*}{ CA } & \multicolumn{3}{|c|}{ TNZ-PABA } & \multicolumn{3}{|c|}{ TNZ-SA } & \multicolumn{2}{|c|}{ TNZ-CA } \\
\hline & & & & & PM & LAG & $\begin{array}{c}\text { CRYS- } \\
\text { ACE }\end{array}$ & PM & LAG & CRYS-етон & PM & LAG \\
\hline 1 & 155.3 & - & - & - & 155.4 & 152.3 & 152.2 & 155.3 & 152.7 & 152.7 & 155.3 & 152.7 \\
\hline 2 & 135.0 & - & - & - & 135.1 & 134.2 & 134.2 & 135.0 & 135.0 & 135.0 & 135.0 & 133.3 \\
\hline 3 & 138.3 & - & - & - & 138.3 & 136.9 & 136.8 & 138.3 & 138.3 & 138.3 & 138.3 & 137.1 \\
\hline 4 & 39.5 & - & - & - & 39.6 & 40.3 & 40.2 & 39.5 & 38.6 & 38.6 & 39.5 & 40.5 \\
\hline 5 & 47.0 & - & - & - & 47.0 & 48.6 & 48.8 & 47.0 & 52.0 & 52.1 & 47.0 & 48.9 \\
\hline 6 & 47.0 & - & - & - & 47.0 & 46.5 & 46.7 & 47.0 & 48.5 & 48.5 & 47.0 & 47.7 \\
\hline 7 & 8.8 & - & - & - & 8.9 & 8.0 & 7.0 & 8.8 & 5.0 & 5.0 & 8.8 & 8.8 \\
\hline 8 & 15.9 & - & - & - & $16.0 / 13.9^{a}$ & 13.9 & 13.6 & 15.9 & 13.6 & 13.6 & 15.9 & 14.0 \\
\hline 9 & - & 116.7 & - & - & $116.6 / 119.5^{a}$ & 119.6 & 119.4 & - & - & - & - & - \\
\hline 10 & - & 131.2 & - & - & $131.1 / 133.0^{a}$ & 133.0 & 132.9 & - & - & - & - & - \\
\hline 11 & - & 115.7 & - & - & 115.7 & 114.2 & 114.0 & - & - & - & - & - \\
\hline 12 & - & 152.9 & - & - & 152.7 & 154.1 & 154.2 & - & - & - & - & - \\
\hline 13 & - & 112.1 & - & - & $112.3 / 113.2^{a}$ & 113.1 & 113.1 & - & - & - & - & - \\
\hline 14 & - & 131.2 & - & - & $131.1 / 133.0^{a}$ & 133.0 & 133.0 & - & - & - & - & - \\
\hline 15 & - & 174.2 & - & - & $174.2 / 170.2^{a}$ & 170.3 & 170.2 & - & - & - & - & - \\
\hline 16 & - & - & 112.2 & - & - & - & - & 112.2 & 113.9 & 114.0 & - & - \\
\hline
\end{tabular}




\begin{tabular}{|c|c|c|c|c|c|c|c|c|c|c|c|c|}
\hline 17 & - & - & 162.3 & - & - & - & - & 162.3 & 163.3 & 163.3 & - & - \\
\hline 18 & - & - & 118.5 & - & - & - & - & 118.5 & 117.0 & 117.0 & - & - \\
\hline 19 & - & - & 138.7 & - & - & - & - & 138.7 & 135.6 & 135.6 & - & - \\
\hline 20 & - & - & 121.3 & - & - & - & - & 121.3 & 119.0 & 119.0 & - & - \\
\hline 21 & - & - & 133.4 & - & - & - & - & 133.4 & 129.5 & 129.6 & - & - \\
\hline 22 & - & - & 176.3 & - & - & - & - & 176.3 & 170.6 & 170.6 & - & - \\
\hline 23 & - & - & - & 174.9 & - & - & - & - & - & - & 174.9 & 173.9 \\
\hline 23' & - & - & - & 176.4 & - & - & - & - & - & - & 176.4 & 173.9 \\
\hline 24 & - & - & - & 43.1 & - & - & - & - & - & - & 43.1 & 43.1 \\
\hline 24 & - & - & - & 44.1 & - & - & - & - & - & - & 44.1 & 44.6 \\
\hline 25 & - & - & - & 72.1 & - & - & - & - & - & - & 72.1 & 74.0 \\
\hline 26 & - & - & - & 179.4 & - & - & - & - & - & - & 179.4 & 178.9 \\
\hline+ & - & - & - & - & - & - & 18.0 & - & - & - & - & - \\
\hline$\#$ & - & - & - & - & - & 30.7 & 30.7 & - & - & - & - & - \\
\hline+ & - & - & - & - & - & - & 58.0 & - & - & - & - & - \\
\hline
\end{tabular}

a Low intensity signal, indicating the presence of a minor quantity of TNZ-PABA cocrystal in the PM, which was formed during the NMR experiment. + Carbon atoms of ethanol. \# Carbon atoms of aceton 
Table S5

\begin{tabular}{ll}
\hline Sample $^{\mathbf{a}}$ & $\mathbf{p H}$ \\
\hline TNZ-PABA LAG & 3.5 \\
TNZ-SA LAG & 3.4 \\
TNZ-CA LAG & 3.0 \\
MilliQ ${ }^{\circledR}$ water & 5.5
\end{tabular}

a pKa values: $\mathrm{PABA}=2.38$ (amine) and 4.85 (carboxylic acid) at $25^{\circ} \mathrm{C}$ (PubChem); $\mathrm{SA}=2.98$ (carboxylic acid, PubChem); $\mathrm{CA}=$ 2.79 (PubChem); SUC $=4.207$ (pKa1) and 5,635 (pKa2) at $25^{\circ} \mathrm{C}$ (PubChem), and NA $=3.35$ (conjugate acid) at $20^{\circ} \mathrm{C}$ (PubChem). ${ }^{b}$ For TNZ not coincident $p K a$ values were found in the literature: 4.7 (PubChem); 2.3 (DrugBank), and 1.82 (J Pharm Sci. 2003;92:739-46, DOI: 10.1002/jps.10349; Pharmazie 2007; 62:327-36, doi: 10.1691/ph.2007.5.6111). 